\title{
Changes in Serum Dickkopf-1, RANK Ligand, Osteoprotegerin, and Bone Mineral Density after Allogeneic Hematopoietic Stem Cell Transplantation Treatment
}

\author{
Eunhee Jang ${ }^{1}$, Jeonghoon $\mathrm{Ha}^{2}$, Ki-Hyun Baek ${ }^{3}$, Moo Il Kang ${ }^{2}$ \\ ${ }^{1}$ Division of Endocrinology, Department of Internal Medicine, Mizmedi Hospital; ${ }^{2}$ Division of Endocrinology and Metabolism, \\ Department of Internal Medicine, Seoul St. Mary's Hospital, College of Medicine, The Catholic University of Korea; ${ }^{3}$ Division \\ of Endocrinology and Metabolism, Department of Internal Medicine, Yeouido St. Mary's Hospital, College of Medicine, The \\ Catholic University of Korea, Seoul, Korea
}

Background: Dickkopf-1 (DKK1) regulates bone formation by inhibiting canonical Wnt/ $\beta$-catenin pathway signaling, and indirectly enhances osteoclastic activity by altering the expression ratio of receptor activator of nuclear factor- $\mathrm{KB}$ ligand (RANKL) relative to osteoprotegerin (OPG). However, it is difficult to explain continued bone loss after allogeneic stem cell transplantation (allo-SCT) in terms of changes in only RANKL and OPG. Few studies have evaluated changes in DKK1 after allo-SCT.

Methods: We prospectively enrolled 36 patients with hematologic malignancies who were scheduled for allo-SCT treatment. Serum DKK1, OPG, and RANKL levels were measured before (baseline), and at 1, 4, 12, 24, and 48 weeks after allo-SCT treatment. Bone mineral density (BMD) was assessed using dual-energy X-ray absorptiometry before (baseline) and 24 and 48 weeks after allo-SCT treatment.

Results: After allo-SCT treatment, the DKK1 level decreased rapidly, returned to baseline during the first 4 weeks, and remained elevated for 48 weeks $(P<0.0001$ for changes observed over time). The serum RANKL/OPG ratio peaked at 4 weeks and then declined $(P<0.001$ for changes observed over time). BMD decreased relative to the baseline at all timepoints during the study period, and the lumbar spine in female patients had the largest decline $(-11.3 \% \pm 1.6 \%$ relative to the baseline at 48 weeks, $P<0.05)$.

Conclusion: Serum DKK1 levels rapidly decreased at 1 week and then continued to increase for 48 weeks; bone mass decreased for 48 weeks following engraftment in patients treated with allo-SCT, suggesting that DKK1-mediated inhibition of osteoblast differentiation plays a role in bone loss in patients undergoing allo-SCT.

Keywords: Bone density; Hematopoietic stem cell transplantation; DKK1; RANK ligand; Osteoprotegerin

\section{INTRODUCTION}

Loss of bone mass after allogeneic hematopoietic stem cell transplantation (allo-SCT) treatment is a common complication

Received: 2 September 2021, Revised: 22 October 2021,

Accepted: 1 November 2021

Corresponding author: Moo Il Kang

Division of Endocrinology and Metabolism, Department of Internal Medicine, Seoul St. Mary's Hospital, College of Medicine, The Catholic University of Korea, 222 Banpo-daero, Seocho-gu, Seoul 06591, Korea

Tel: +82-2-2258-6006, Fax: +82-2-599-3589, E-mail: mikang@catholic.ac.kr that is characterized by a paradoxical uncoupling of impaired bone formation with increased bone resorption, which is reflected by markers of bone turnover [1-4]. Although the underlying mechanisms are not completely understood, osteocalcin is sup-

\section{Copyright $(92021$ Korean Endocrine Society}

This is an Open Access article distributed under the terms of the Creative Commons Attribution Non-Commercial License (https://creativecommons.org/ licenses/by-nc/4.0/) which permits unrestricted non-commercial use, distribution, and reproduction in any medium, provided the original work is properly cited. 
pressed and C-terminal cross-linking telopeptide of type-1 collagen (CTX) levels increase during the high bone turnover period early after transplantation [1,5]. Furthermore, bone mineral density (BMD) decreases rapidly during the first 6 months after allo-SCT treatment [1,6-8] and remains low for 48 months [8,9].

Dickkopf-1 (DKK1), a protein encoded by the DKK1 gene, binds to low-density lipoprotein receptor-related protein (LRP) $5 / 6$ and inactivates the canonical Wnt/ $\beta$-catenin pathway, thereby suppressing Wnt-mediated osteoblast differentiation [10-12]. Despite the complexity of DKK1 model dynamics, evidence suggests that DKK1 inhibits osteoprotegerin (OPG) secretion and increases serum receptor activator of nuclear factor- $\kappa \mathrm{B}$ ligand (sRANKL) production, thereby shifting the sRANKL/ OPG ratio toward osteoclast activation, which results in increased bone resorption in patients with postmenopausal osteoporosis [10,12,13].

We previously observed altered osteoclast activity after alloSCT treatment and found that the sRANKL/OPG ratio and CTX levels increased immediately after treatment [1-3,5]. Although previous studies have reported a decline in osteocalcin levels immediately after allo-SCT treatment, this evidence is insufficient to explain the bone loss observed due to osteoblast changes that occur immediately after treatment. In previous studies, the occurrence of bone loss after transplantation was mainly studied in terms of osteoclast activity. However, it is difficult to completely explain bone loss that persists after transplantation; therefore, a study evaluating osteoblast activity is needed. DKK1 is a good indicator for indirectly observing osteoblastic activity; however, no studies have simultaneously observed changes in DKK1 and BMD in patients with allo-SCT. Therefore, the purpose of this study was to further investigate changes in DKK1 levels, concurrently with those of sRANKL and OPG, after allo-SCT treatment and to determine their effects on BMD.

\section{METHODS}

\section{Study design}

We prospectively evaluated the changes in DKK1, OPG, and sRANKL levels for 48 weeks after allo-SCT treatment in patients who underwent hematopoietic stem cell transplantation (HSCT). Thirty-six patients (15 female and 21 male) with hematological malignancies awaiting allo-SCT treatment were enrolled and followed for 48 weeks. Exclusion criteria included secondary osteoporosis caused by factors other than hematologic malignancies (e.g., rheumatoid arthritis, Paget disease, primary hyper- parathyroidism, and hyperthyroidism), a history of other treatment that affected bone metabolism during the preceding 3 months (e.g., glucocorticoids, calcitonin, fluoride, sex hormones, other anti-resorptive agents, and parathyroid hormone), elevated serum creatinine $(>1.5 \mathrm{mg} / \mathrm{L}$ in males or $>1.4 \mathrm{mg} / \mathrm{dL}$ in $\mathrm{fe}-$ males), or liver enzymes $\geq 2.5$-fold higher than the upper limit of normal. This study was approved by the Institutional Review Board of Yeouido St. Mary's Hospital (KC10SI-S10114). Written informed consent was obtained from all subjects before their enrollment in the study.

\section{Measurements}

Venous blood was obtained in the morning after an overnight fast, immediately centrifuged to obtain serum, and stored at $-70^{\circ} \mathrm{C}$ until assayed. DKK1, OPG, and sRANKL levels were measured before and 1, 4, 12, 24, and 48 weeks after allo-SCT treatment. DKK1 levels were measured using an enzyme-linked immunosorbent assay (ELISA) (Biomedica, Vienna, Austria; intra-assay coefficient of variation [CV] 7\% to $8 \%$ and inter-assay CV $9 \%$ to $12 \%$ ) on a V-MAX 220 VAC ELISA reader (Molecular Devices, San Jose, CA, USA). OPG and RANKL levels were measured using an ELISA (R\&D Systems, Minneapolis, MN, USA; PeproTech, Rocky Hill, NJ, USA). The BMD of the lumbar spine (lumbar vertebrae L1-L4), total hip, and femoral neck was measured using dual-energy X-ray absorptiometry (Hologic Delphi W, Watham, MA, USA) before and 24 and 48 weeks after allo-SCT treatment. The calculated CV was $1.2 \%$ for the lumbar spine and $1.9 \%$ for the femoral neck.

\section{Transplantation procedure}

The conditioning regimen comprised cyclophosphamide (50 to $60 \mathrm{mg} / \mathrm{kg} /$ day for 2 days; total of 100 to $120 \mathrm{mg} / \mathrm{kg}$ ) with fractionated total-body irradiation (total of 400 to $1,320 \mathrm{cGy}$ ), followed by graft-versus-host disease (GVHD) prophylaxis with tacrolimus or cyclosporine, and a short-term course of methotrexate ( $5 \mathrm{mg} / \mathrm{m}^{2}$ intravenous bolus on days $1,3,6$, and 11). Bone marrow stem cells or granulocyte colony-stimulating factor-mobilized peripheral blood stem cells were administered on day 0 . During the allo-SCT procedures, all patients were treated in a designated room with laminar airflow isolation. General supportive care was provided, including administration of granulocyte colony-stimulating factor, prophylaxis of veno-occlusive disease, and administration of prophylactic antibiotics.

\section{Primary and secondary outcomes of interest}

The primary outcomes of interest were the changes in the serum 
levels of DKK1, sRANKL, OPG, and sRANKL/OPG ratio observed during the 48 weeks after allo-SCT treatment. The secondary outcomes of interest were the changes in areal BMD of the lumbar spine, femoral neck, and total hip 24 and 48 weeks after allo-SCT treatment. Changes in the percentage of BMD from baseline were also investigated.

\section{Statistical analysis}

Serum DKK1, sRANKL, OPG, and BMD levels were analyzed using repeated-measures analysis of variance (ANOVA) to determine the significance of the changes observed over the timelines indicated. All $P$ values were two-sided; the level of significance was $<0.05$. All values are based on $95 \%$ confidence intervals and are presented as the mean \pm standard error or number (\%). All statistical analyses were performed using SPSS for Windows version 24.0 (IBM Corp., Armonk, NY, USA). Graphics were produced using GraphPad Prism version 5.0 (GraphPad Software Inc., San Diego, CA, USA).

\section{RESULTS}

\section{Baseline characteristics}

The clinical and biochemical data obtained from 36 patients are summarized in Table 1. The mean patient age was $36.0 \pm 1.8$ years (35.4 \pm 2.4 years for male subjects and $36.7 \pm 2.5$ years for female subjects). The mean body mass index was $23.7 \pm 0.8 \mathrm{~kg} / \mathrm{m}^{2}$ for male subjects and $21.2 \pm 0.8 \mathrm{~kg} / \mathrm{m}^{2}$ for female subjects. The most common underlying malignancy was acute lymphocyte leukemia $(33.3 \%)$. All patients received total-body irradiation and were treated with immunosuppressants as prophylactic treatment for GVHD. The serum levels of DKK1, sRANKL, and OPG were $164.4 \pm 25.9,1,352.6 \pm 550.1$, and $563.9 \pm 94.1 \mathrm{pg} / \mathrm{mL}$, respectively. The mean BMD for all patients was $0.941 \pm 0.164 \mathrm{~g} / \mathrm{cm}^{2}$ for the lumbar spine, $0.816 \pm 0.129 \mathrm{~g} / \mathrm{cm}^{2}$ for the femoral neck, and $0.819 \pm 0.094 \mathrm{~g} / \mathrm{cm}^{2}$ for the total hip.

Changes in serum DKK1 levels and the RANKL/OPG ratio Fig. 1 shows the changes in the serum levels of DKK1, sRANKL,

\begin{tabular}{|c|c|c|c|}
\hline Clinical parameter & All subjects $(n=36)$ & Male subjects $(n=21)$ & Female subjects $(n=15)$ \\
\hline Age, yr & $36.0 \pm 1.8$ & $35.4 \pm 2.4$ & $36.7 \pm 2.5$ \\
\hline Body mass index, $\mathrm{kg} / \mathrm{m}^{2}$ & $22.2 \pm 0.6$ & $23.7 \pm 0.8$ & $21.2 \pm 0.8$ \\
\hline \multicolumn{4}{|l|}{ Hematologic disease } \\
\hline Acute myeloid leukemia & $11(30.6)$ & $7(33.3)$ & $4(26.7)$ \\
\hline Acute lymphoblastic leukemia & $12(33.3)$ & $7(33.3)$ & $5(33.3)$ \\
\hline Severe aplastic anemia & $4(11.1)$ & $2(9.5)$ & $1(6.7)$ \\
\hline Myelodysplastic syndrome & $6(16.7)$ & $3(14.4)$ & $2(13.3)$ \\
\hline Others & $3(8.3)$ & $2(9.5)$ & $3(20.0)$ \\
\hline Steroid exposure after transplantation & $25(69.4)$ & $15(71.4)$ & $10(66.7)$ \\
\hline Immunosuppressant for GVHD prophylaxis & $36(100)$ & $21(100)$ & $15(100)$ \\
\hline Total-body irradiation & $36(100)$ & $21(100)$ & $15(100)$ \\
\hline Acute GVHD (grade III-IV) & $21(58.3)$ & $12(57.1)$ & $9(60.0)$ \\
\hline Chronic GVHD (moderate to severe) & $20(55.6)$ & $12(57.1)$ & $8(53.0)$ \\
\hline \multicolumn{4}{|l|}{ Serum level, $\mathrm{pg} / \mathrm{mL}$} \\
\hline DKK1 & $164.4 \pm 25.9$ & $190.4 \pm 31.2$ & $138.3 \pm 22.7$ \\
\hline sRANKL & $1,352.6 \pm 550.1$ & $1,833.6 \pm 412.3$ & $871.2 \pm 301.5$ \\
\hline OPG & $563.9 \pm 94.1$ & $615.2 \pm 101.3$ & $512.7 \pm 87.6$ \\
\hline \multicolumn{4}{|l|}{ Bone mineral density, $\mathrm{g} / \mathrm{cm}^{2}$} \\
\hline Lumbar spine & $0.941 \pm 0.164$ & $0.912 \pm 0.174$ & $0.972 \pm 0.147$ \\
\hline Femoral neck & $0.816 \pm 0.129$ & $0.850 \pm 0.132$ & $0.782 \pm 0.118$ \\
\hline Total hip & $0.819 \pm 0.094$ & $0.858 \pm 0.127$ & $0.779 \pm 0.164$ \\
\hline
\end{tabular}

Values are expressed as mean \pm standard deviation or number (\%).

GVHD, graft-versus-host disease; DKK1, dickkopf-1; sRANKL, serum receptor activator of nuclear factor-kB ligand; OPG, osteoprotegerin. 
OPG, and the sRANKL/OPG ratio during the study period. Serum DKK1 levels decreased abruptly immediately after allo-SCT treatment, recovered to baseline at about 4 weeks, and subsequently increased consistently over the remaining weeks studied $(P<0.0001$ for changes observed over time) (Fig. 1A). Serum sRANKL levels increased progressively until 4 weeks after alloSCT treatment and then declined to baseline after 12 weeks, remaining low for the remainder of the study $(P<0.0001$ for changes observed over time) (Fig. 1B). Serum OPG levels increased immediately after allo-SCT treatment (1 week post-allo-SCT treatment) and then declined (Fig. 1C). The sRANKL/ OPG ratio declined immediately after allo-SCT treatment, peaked at 4 weeks, and then declined progressively for the remainder of the study $(P<0.0001$ for changes observed over time) (Fig. 1D). This result was consistent even when several clinical parameters were adjusted. DKK1 levels were higher in men than in women during the study period, and the sRANKL and OPG levels and sRANKL/OPG ratio were all higher in men than in women except at 4 weeks (Fig. 1, dotted blue line for male subjects and red line for female subjects).

\section{Changes in BMD after allo-SCT treatment}

The changes in BMD relative to baseline at 24 and 48 weeks after allo-SCT treatment were analyzed (Fig. 2). In the entire study population, BMD decreased relative to the baseline at all skeletal sites at both 24 and 48 weeks $(P<0.05)$, and this change was also significant in the subgroup analysis according to sex (Table 2). A greater reduction in BMD relative to baseline was observed in women than in men at all measurement sites at both timepoints. The lumbar spine BMD decreased by $6.2 \% \pm 2.9 \%$ $(P<0.05)$ in men and $11.3 \% \pm 1.6 \%(P<0.05)$ in women relative to baseline at 48 weeks post-treatment (Fig. 2A). The femo-
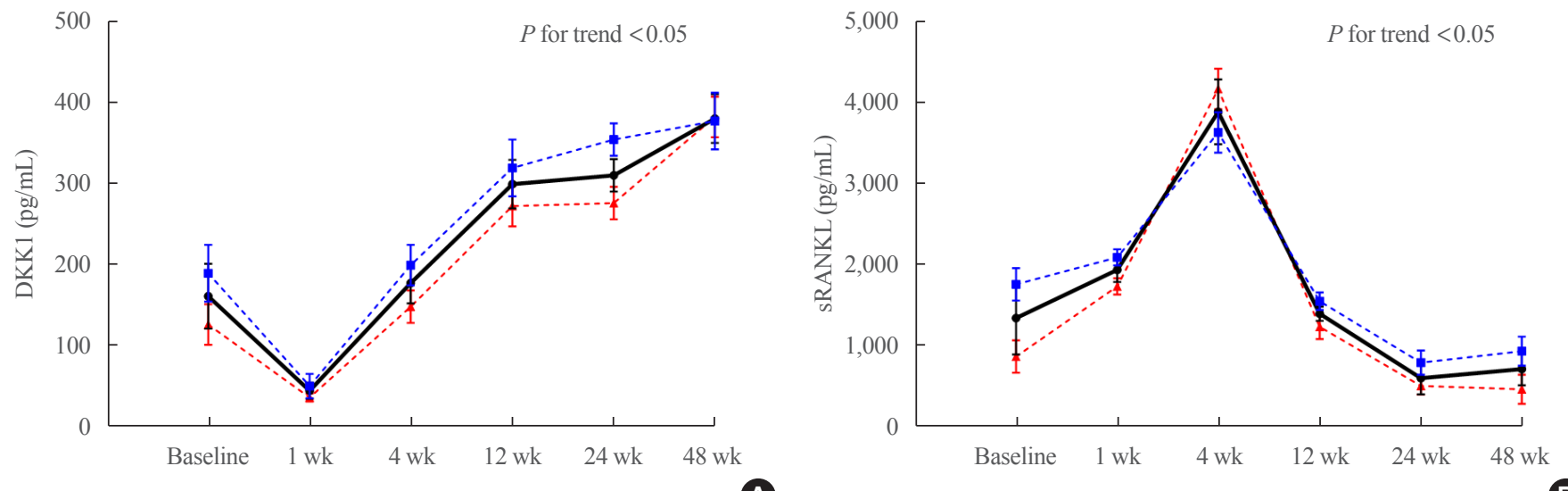

A
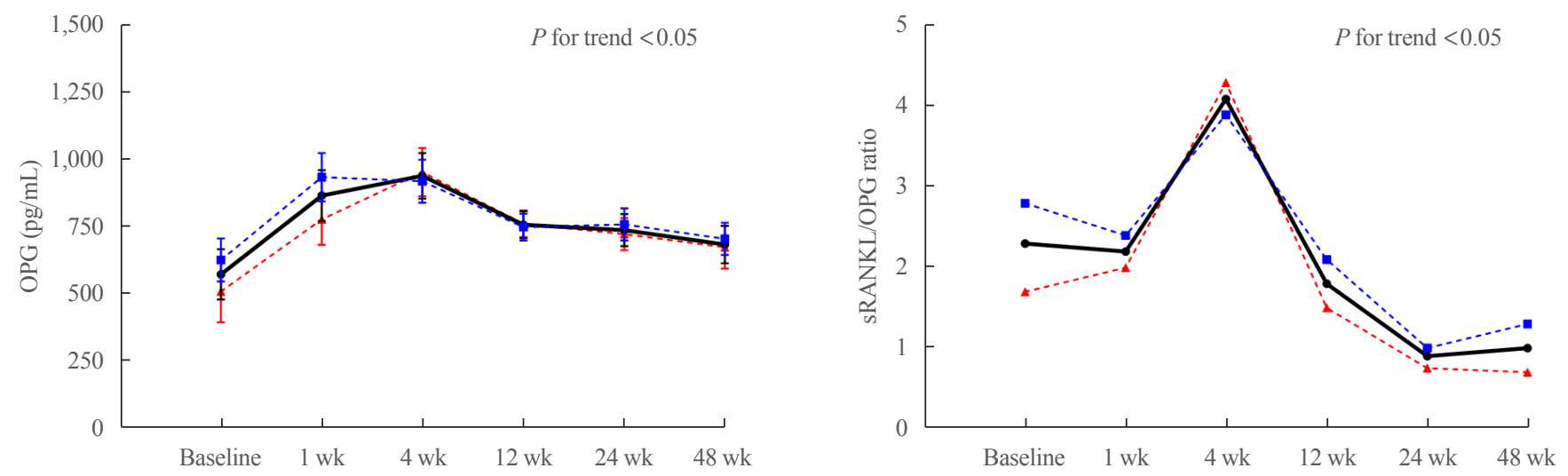

C

Fig. 1. Changes in the serum levels of (A) dickkopf-1 (DKK1), (B) serum receptor activator of nuclear factor- $\kappa \mathrm{B}$ ligand (sRANKL), (C) osteoprotegerin $(\mathrm{OPG})$, and (D) the receptor activator of nuclear factor- $\mathrm{kB}$ ligand (RANKL)/OPG ratio before and after allogeneic stem cell transplantation treatment. Data are presented as the mean \pm standard deviation. Solid line, all subjects; blue dotted line, male subjects; red dotted line, female subjects. 
ral neck BMD decreased by $8.8 \% \pm 4.8 \%(P<0.05)$ in men and $10.9 \% \pm 4.2 \%(P<0.05)$ in women relative to baseline at 48 weeks post-treatment (Fig. 2B). For the total hip, significant bone loss was detected relative to baseline at 48 weeks posttreatment $(-7.3 \% \pm 2.0 \%, P<0.05$ in men and $-7.6 \% \pm 3.4 \%$, $P<0.05$ in women) (Fig. 2C). The greatest decreases in BMD relative to baseline were observed in the femoral neck in men at
48 weeks and in the lumbar spine in women at 48 weeks. Regression analysis showed that the DKK1 change at week 48 showed a significant inverse correlation with lumbar spine BMD at week 48 in the entire study population $(\beta=-0.0366$, $P<0.05)$. Changes in DKK1 levels measured at different periods and the associations with BMD all showed inverse correlations, but these were not statistically significant.
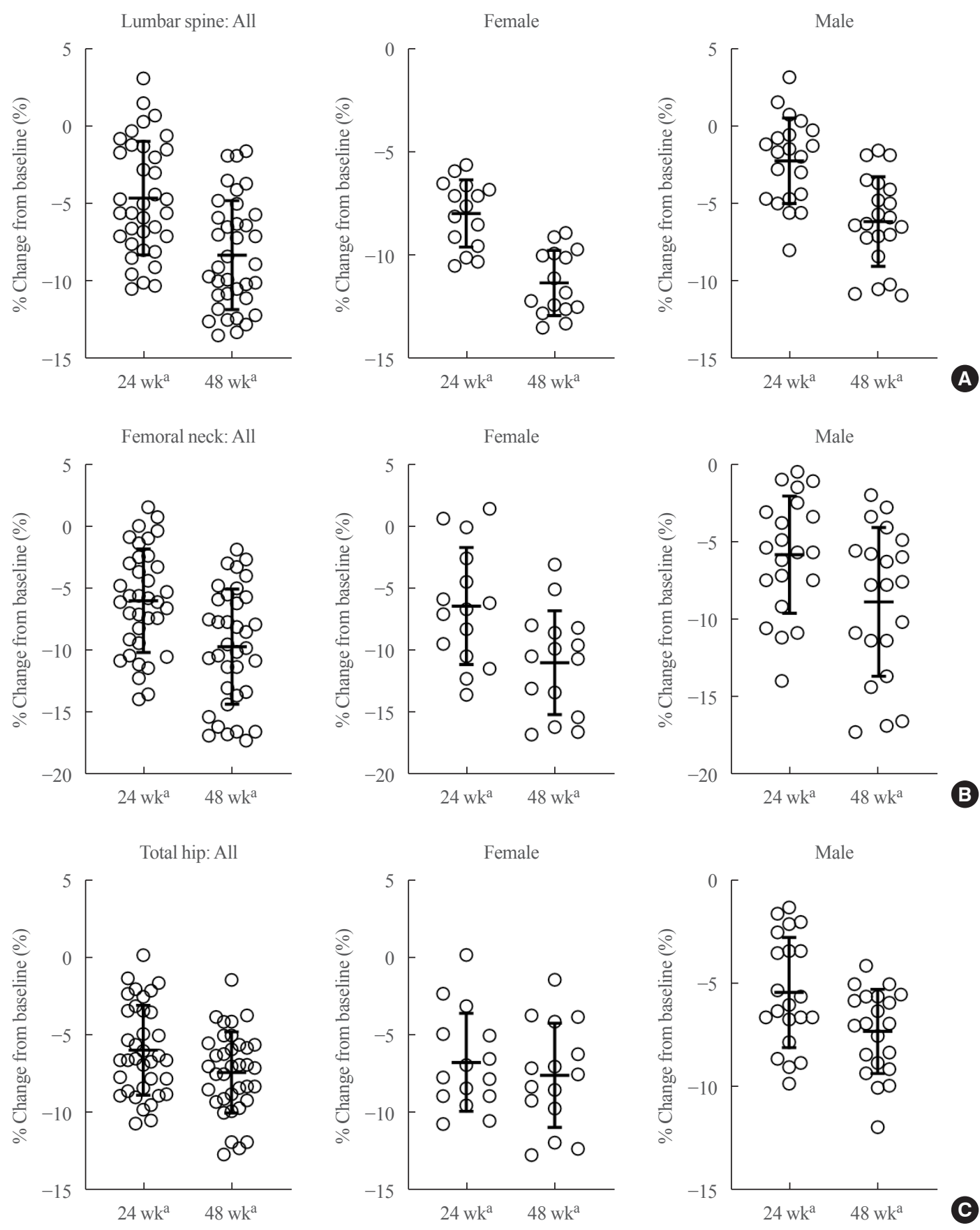

Fig. 2. Changes in bone mineral density at each measurement site relative to baseline. (A) Lumbar spine, (B) femoral neck, (C) total hip. ${ }^{a} P<0.05$ relative to baseline. 


\begin{tabular}{|c|c|c|c|}
\hline & Lumbar spine & Femoral neck & Total hip \\
\hline \multicolumn{4}{|l|}{ All subjects } \\
\hline 24 weeks & $-4.6 \pm 3.6$ & $-6.0 \pm 4.1$ & $-6.0 \pm 2.9$ \\
\hline 48 weeks & $-8.3 \pm 3.5$ & $-9.7 \pm 4.6$ & $-7.4 \pm 2.6$ \\
\hline \multicolumn{4}{|c|}{ Male subjects } \\
\hline 24 weeks & $-2.3 \pm 2.7$ & $-5.7 \pm 3.8$ & $-5.4 \pm 2.7$ \\
\hline 48 weeks & $-6.2 \pm 2.9$ & $-8.8 \pm 4.8$ & $-7.3 \pm 2.0$ \\
\hline \multicolumn{4}{|c|}{ Female subjects } \\
\hline 24 weeks & $-8.0 \pm 1.6$ & $-6.4 \pm 4.7$ & $-6.7 \pm 3.2$ \\
\hline 48 weeks & $-11.3 \pm 1.6$ & $-10.9 \pm 4.2$ & $-7.6 \pm 3.4$ \\
\hline
\end{tabular}

\section{DISCUSSION}

In this prospective study of 36 patients who underwent alloSCT treatment, serum DKK1 levels increased progressively for 48 weeks after treatment, whereas the sRANKL/OPG level steadily declined after peaking at 4 weeks. Areal BMD continued to decrease at all measurement sites during the 48 weeks monitored after allo-SCT treatment. The femoral neck was the site with the largest decline in male patients, and the lumbar spine was the site with the largest decline in female patients.

DKK1, a soluble inhibitor of the canonical Wnt/ $\beta$-catenin pathway, inhibits the differentiation of mesenchymal stem cells into osteoblasts $[10,14]$. Reduced DKK1 expression induces an increase in trabecular and cortical bone mass in vivo [15]. Li et al. [16] reported that DKK1 directly influences bone formation in vivo via dose-dependent suppression of osteoblast matrix mineralization. DKK1 is involved in several bone diseases [14], such as glucocorticoid-induced osteoporosis [17,18], ovariectomy-associated osteoporosis [19], and postmenopausal osteoporosis [20-22].

We previously suggested that bone marrow stromal cells are of recipient origin and that delayed osteoblast differentiation in recipient bone marrow is the cause of bone loss after SCT [5]. We also investigated changes in the bone turnover markers CTX and osteocalcin, monitored changes in osteoclast activity by measuring serum RANKL and OPG levels, and concluded that bone loss occurs after allo-SCT treatment $[1,2]$. However, the changes in RANKL and OPG favoring bone loss occurred early (within 3 months after allo-SCT treatment), which is not sufficient to explain the continuing bone loss observed up to 1 year after allo-SCT treatment. Therefore, in this study, we sought to determine the mechanisms underlying the observed bone loss after allo-SCT treatment by investigating the changes in DKK1, which is presumed to be of osteocyte origin and influences osteoblast differentiation, and changes in the levels of RANKL and $\mathrm{OPG}$, which play roles in osteoclast activity.

In the present study, serum DKK1 levels progressively increased for 48 weeks after allo-SCT treatment. This persistent increase in DKK1 levels suggests that progressive impairment of bone formation occurs, which provides an explanation for the sustained bone loss observed after allo-SCT treatment. BMD decreased rapidly for the first 24 weeks following allo-SCT treatment and continued to decrease for the remainder of the study. This decrease can be attributed to an initial synergistic effect between the increasing levels of DKK1 and the RANKL/ OPG ratio. Subsequently, the RANKL/OPG ratio gradually decreased, whereas DKK1 levels continued to increase, suggesting that bone loss continued to occur even if the observed differences in bone loss were not as significant. The BMD decrease in the femoral neck and total hip was significant during the study period. We previously reported that bone loss occurring after transplantation was more pronounced in the femur than in the lumbar spine [1]. Kang et al. [1] reported that 12 months after transplantation, lumbar BMD decreased by $2.2 \%$ and femoral BMD decreased by $6.2 \%$, which is in agreement with our results.

Based on the observations of increased osteoclast activity, as reflected by changes in RANKL and OPG, the current recommendation for the prevention and treatment of bone loss after a patient receives HSCT is bisphosphonate administration to inhibit osteoclast activity [23-25]. Administration of denosumab, another potent anti-resorptive agent [26], is also a possible option [27]. However, there are conflicting results regarding the effect of bisphosphonates on the level of RANKL/OPG. Vega et al. [28] reported that levels of RANKL/OPG were not affected by bisphosphonates. It is unknown whether bisphosphonates effectively regulate the mechanisms underlying the DKK1-mediated inhibition of osteoblast differentiation. Studies evaluating the effects of anti-resorptive agents on DKK1 levels and the prevention and treatment of bone loss after transplantation have yet to be presented in the literature. Mechanical loading is also an important intervention in bone formation [29,30], and compressive loading increases the anabolic bone response in DKK1knockout mice [31]. Based on our findings that DKK1 levels continue to increase after allo-SCT treatment, increasing physical activity and ensuring a sufficient mechanical load after allo- 
SCT treatment, thereby increasing osteoblast differentiation, may have important clinical implications associated with treatment regimens that include anti-resorptive agents.

There are several limitations of this study to note. First, many complex and dynamic factors affect bone metabolism after alloSCT treatment; therefore, we could not determine the specific cause underlying the changes in DKK1 levels observed in this study. Second, it is difficult to conclude that all changes in DKK1 levels are related to osteoblast differentiation. In addition, simultaneous measurements of bone turnover markers or sclerostin, associated with osteoblast differentiation and activity, are missing. Because multiple factors play a role in osteoblast differentiation, the deterioration in bone formation observed cannot be attributed only to changes in DKK1 levels. Third, DKK1, sRANKL, and OPG levels were evaluated in only the serum, and not in the bone marrow. Therefore, we cannot exclude any effects on the bone marrow per se. An additional limitation of the study is the small sample size and the lack of comparison with age- and sex-matched controls.

In conclusion, serum DKK1 levels persistently increased following engraftment in patients treated with allo-SCT over the 48 weeks studied. To the best of our knowledge, this is the first study that investigated the changes in DKK1 levels in patients undergoing allo-SCT treatment and concurrently monitored changes in BMD. Although additional longitudinal studies are needed to elucidate a clear link between DKK1 levels and bone loss after allo-SCT treatment, it is apparent that DKK1-mediated inhibition of osteoblast differentiation plays a role in bone loss observed in patients treated with allo-SCT.

\section{CONFLICTS OF INTEREST}

No potential conflict of interest relevant to this article was reported.

\section{AUTHOR CONTRIBUTIONS}

Conception or design: K.H.B., M.I.K. Acquisition, analysis, or interpretation of data: E.J., J.H. Drafting the work or revising: E.J., J.H. Final approval of the manuscript: M.I.K.

\section{ORCID}

Eunhee Jang https://orcid.org/0000-0002-4337-4497

Moo Il Kang https://orcid.org/0000-0002-8391-6430

\section{REFERENCES}

1. Kang MI, Lee WY, Oh KW, Han JH, Song KH, Cha BY, et al. The short-term changes of bone mineral metabolism following bone marrow transplantation. Bone 2000;26:275-9.

2. Baek KH, Oh KW, Lee WY, Tae HJ, Rhee EJ, Han JH, et al. Changes in the serum sex steroids, IL-7 and RANKL-OPG system after bone marrow transplantation: influences on bone and mineral metabolism. Bone 2006;39:1352-60.

3. Baek KH, Kang MI. Biomarkers of bone and mineral metabolism following bone marrow transplantation. Adv Clin Chem 2009;49:99-120.

4. Stern JM, Sullivan KM, Ott SM, Seidel K, Fink JC, Longton $\mathrm{G}$, et al. Bone density loss after allogeneic hematopoietic stem cell transplantation: a prospective study. Biol Blood Marrow Transplant 2001;7:257-64.

5. Lee WY, Cho SW, Oh ES, Oh KW, Lee JM, Yoon KH, et al. The effect of bone marrow transplantation on the osteoblastic differentiation of human bone marrow stromal cells. J Clin Endocrinol Metab 2002;87:329-35.

6. Kashyap A, Kandeel F, Yamauchi D, Palmer JM, Niland JC, Molina A, et al. Effects of allogeneic bone marrow transplantation on recipient bone mineral density: a prospective study. Biol Blood Marrow Transplant 2000;6(3A):344-51.

7. Valimaki MJ, Kinnunen K, Volin L, Tahtela R, Loyttyniemi $\mathrm{E}$, Laitinen $\mathrm{K}$, et al. A prospective study of bone loss and turnover after allogeneic bone marrow transplantation: effect of calcium supplementation with or without calcitonin. Bone Marrow Transplant 1999;23:355-61.

8. Weilbaecher KN. Mechanisms of osteoporosis after hematopoietic cell transplantation. Biol Blood Marrow Transplant 2000;6(2A):165-74.

9. Schulte CM, Beelen DW. Bone loss following hematopoietic stem cell transplantation: a long-term follow-up. Blood 2004;103:3635-43.

10. Pinzone JJ, Hall BM, Thudi NK, Vonau M, Qiang YW, Rosol TJ, et al. The role of dickkopf-1 in bone development, homeostasis, and disease. Blood 2009;113:517-25.

11. Schupbach D, Comeau-Gauthier M, Harvey E, Merle G. Wnt modulation in bone healing. Bone 2020;138:115491.

12. Moorer MC, Riddle RC. Regulation of osteoblast metabolism by Wnt signaling. Endocrinol Metab (Seoul) 2018;33: 318-30.

13. Diarra D, Stolina M, Polzer K, Zwerina J, Ominsky MS, Dwyer D, et al. Dickkopf-1 is a master regulator of joint remodeling. Nat Med 2007;13:156-63. 
14. Huang Y, Liu L, Liu A. Dickkopf-1: current knowledge and related diseases. Life Sci 2018;209:249-54.

15. Morvan F, Boulukos K, Clement-Lacroix P, Roman Roman S, Suc-Royer I, Vayssiere B, et al. Deletion of a single allele of the Dkk1 gene leads to an increase in bone formation and bone mass. J Bone Miner Res 2006;21:934-45.

16. Li J, Sarosi I, Cattley RC, Pretorius J, Asuncion F, Grisanti $\mathrm{M}$, et al. Dkk1-mediated inhibition of Wnt signaling in bone results in osteopenia. Bone 2006;39:754-66.

17. Hurson CJ, Butler JS, Keating DT, Murray DW, Sadlier DM, O’Byrne JM, et al. Gene expression analysis in human osteoblasts exposed to dexamethasone identifies altered developmental pathways as putative drivers of osteoporosis. BMC Musculoskelet Disord 2007;8:12.

18. Thiele S, Ziegler N, Tsourdi E, De Bosscher K, Tuckermann JP, Hofbauer LC, et al. Selective glucocorticoid receptor modulation maintains bone mineral density in mice. J Bone Miner Res 2012;27:2242-50.

19. Wang FS, Ko JY, Lin CL, Wu HL, Ke HJ, Tai PJ. Knocking down dickkopf-1 alleviates estrogen deficiency induction of bone loss: a histomorphological study in ovariectomized rats. Bone 2007;40:485-92.

20. Jemtland R, Holden M, Reppe S, Olstad OK, Reinholt FP, Gautvik VT, et al. Molecular disease map of bone characterizing the postmenopausal osteoporosis phenotype. J Bone Miner Res 2011;26:1793-801.

21. Reppe S, Refvem H, Gautvik VT, Olstad OK, Hovring PI, Reinholt FP, et al. Eight genes are highly associated with $\mathrm{BMD}$ variation in postmenopausal Caucasian women. Bone 2010;46:604-12.

22. Fassio A, Adami G, Benini C, Vantaggiato E, Saag KG, Giollo A, et al. Changes in Dkk-1, sclerostin, and RANKL se- rum levels following discontinuation of long-term denosumab treatment in postmenopausal women. Bone 2019; 123:191-5.

23. Majhail NS, Rizzo JD, Lee SJ, Aljurf M, Atsuta Y, Bonfim C, et al. Recommended screening and preventive practices for long-term survivors after hematopoietic cell transplantation. Biol Blood Marrow Transplant 2012;18:348-71.

24. Kendler DL, Body JJ, Brandi ML, Broady R, Cannata-Andia J, Cannata-Ortiz MJ, et al. Bone management in hematologic stem cell transplant recipients. Osteoporos Int 2018; 29:2597-610.

25. McClune BL, Majhail NS. Osteoporosis after stem cell transplantation. Curr Osteoporos Rep 2013;11:305-10.

26. Brown JP. Long-term treatment of postmenopausal osteoporosis. Endocrinol Metab (Seoul) 2021;36:544-52.

27. Jeong C, Kim HJ, Lee S, Kang MI, Ha J. Effect of denosumab on bone mineral density of hematopoietic stem cell transplantation recipients. Int J Endocrinol 2020;2020: 3410921.

28. Vega D, Maalouf NM, Sakhaee K. Clinical review \#: the role of receptor activator of nuclear factor-kappaB (RANK)/ RANK ligand/osteoprotegerin: clinical implications. J Clin Endocrinol Metab 2007;92:4514-21.

29. Boyle WJ, Simonet WS, Lacey DL. Osteoclast differentiation and activation. Nature 2003;423:337-42.

30. Boppart MD, Kimmel DB, Yee JA, Cullen DM. Time course of osteoblast appearance after in vivo mechanical loading. Bone 1998;23:409-15.

31. Morse A, Ko FC, McDonald MM, Lee LR, Schindeler A, van der Meulen $\mathrm{MC}$, et al. Increased anabolic bone response in Dkk1 KO mice following tibial compressive loading. Bone 2020;131:115054. 